\title{
Ueber das System der Cometen 1843 I, 1880 I und 1882 II.
}

Der vorstehende Artikel von Dr. M. W. Meyer veraalasst mich aus einer noch nicht beendeten Untersuchung iiber das genannte Cometensystem diejenigen Resultate hier kurz mitzutheilen, welche die Bahn des Cometen r880 I betreffen.

Mit Zugrundelegung der Elemente von Gould (1. N. 2308) versuchte ich zunächst die Beobachtungen durch eine Parabel darzustellen, wobei sich zeigte, dass nur 4 Elemente sich als Functionen des funften mit hinreict.ender (ienauigkeit bestimmen liessen. Das fünfte Element, für welches man passender Weise den Knoten nehmen kann, bleibt innerhalb ziemlich weiter Grenzen willkürlich, und je nach dessen Annahme kann man recht verschiedenartige Elemente er. halten.

Mit dieser Einschränkung darf ich wohl die folgende Parabel als definitive Bahn des Cometen 1880 I gelten lassen:

$$
\left.\begin{array}{rl}
T & =1880 \text { Jan. } 27.655734 \text { M. Z. Berlin } \\
\omega & =86^{\circ} \text { 1 } 8^{\prime} 7^{\prime \prime} 30 \\
\delta & =6 \text { 10 } 29.21 \\
i & =144394^{2.31}
\end{array}\right\} \text { M. Aequ. I } 880.0
$$

welche die in 5 Normalörter zusammengefassten Beobachtungen im Sinne $(B-R)$ folgendermassen darstellt:

\begin{tabular}{r|c|c}
\hline \multicolumn{1}{c|}{1880} & $\Delta \alpha \cos \delta$ & $\Delta \delta$ \\
\hline Febr. 6.5 & -0.01 & +3.7 \\
1 0.0 & -0.09 & -0.6 \\
1 3.0 & +0.13 & -1.2 \\
I 5.5 & +0.09 & -1.9 \\
I 8.5 & -0.80 & +3.8
\end{tabular}

Lässt man im letzten Normalort die sehr stark abweichende Rectascension der Cordobaer Beobachtung vom I7. Febr. weg, so sinkt der Fehler auf -0.53 . Die Darstellung ist eine weit bessere, als man bei der Ungenauigkeit der Beobachtungen von vorn herein $\mathrm{zu}$ erwarten berechtigt war.
Dass man bei der Unsicherheit; mit der sich schon die Parabel bestimmt, nicht noch an eine Bestimmung der Excentricität denken darf, ist selbstverständlich. Wie weit man z. P. letztere variiren kann, ohne mit den Beobachtun. gen in Widerspruch zu gerathen, zeigt die in A. N. 2568 von Dr. von Rebeur-Paschwitz mitgetheilte Bahn, welche, obwohl ihr eine Umlaufszeit von $17^{\mathrm{a}} \cdot \mathbf{4}^{86} \mathrm{zu}$ Grunde liegt, immerhin noch einen erträglichen Anschluss an die Beobachtungen zeigt.

Durch das soeben erwähnte muss der Glaube an eine Identität der Cometen I 843 I und I 880 I stark erschüttert werden, und möchte ich mich im Gegentheil der u. a. von Schönfeld in der Sitzung der niederrhein. Gesellschaft für Natur und Heilkunde am 4. Dec. I 882 ausgesprochenen Ansicht zuwenden, dass die beiden genannten Cometen nicht identisch sind, sondern gemeinsam mit dem Cometen 1882 II ein System cometarischer Massen bilden, welches als ursprünglich vereint $z u$ denken ist. Unter diesen Umständen gewinnt die Sicherheit, mit welcher man die Umlaufszeit des Cometen 1 882 II, beiläufig 800 Jahre, berechnen kann, ein erhöhtes Interesse und es ist nur zu hoffen, dass sich eine ähnliche Sicherheit bei der definitiven Bahnberechnung des Cometen 1843 I herausstellen möge; nach den Untersuchungen von Hubbard ist diese Hoffnung allerdings eine geringe.

Es ist nun an sich nicht unwahrscheinlich, dass die 3 genannten Cometen nicht die einzigen Glieder des Systems sind und dass noch andere Theile desselben theils sichtbar gewesen sind, theils uns noch sichtbar werden können. Unter den unvollständig beobachteten Cometen der letzten Jahrzehnte sind zwei, welche möglicherweise dem genannten System angehören können, einmal der im Vorstehenden von Dr. Meyer näher untersuchte Sonnenfinsterniss-Comet 1882 Mai 16 und dann der vielgenannte Comet Pogson vom Jahre 1872. Von dem letzteren hat Bruhns in seiner Untersuchung über die Identität desselben mit dem Biela'schen Cometen (V.J.S. der A. G. Bd. Io S. r6) unter plausiblen Annahmen des Verhältnisses der Entfernungen die folgenden hypothetischen Bahnen abgeleitet:

$$
\begin{aligned}
& \text { Hyp. I } \frac{A_{3}}{A_{1}}=0.99 \\
& \text { Comet Pogson }\left\{\begin{aligned}
T & =1872 \text { Dec. I } 5.4 \text { I } 35 \mathrm{I} \\
\omega & =60^{\circ} 42^{\prime} 34^{\prime \prime} 7 \\
\delta & =331059.9 \\
i & =148475.3 \\
\log q & =8.546599
\end{aligned}\right.
\end{aligned}
$$

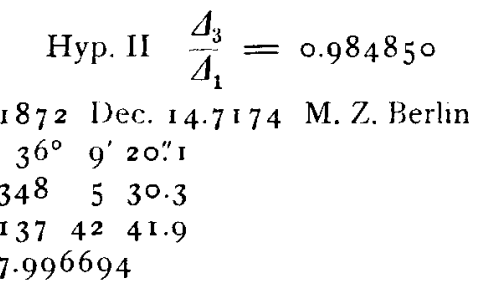

Eine gewisse Aehnlichkeit mit den zu unserem System gehörigen Bahnen ist nicht zu verkennen, doch kann erst eine nähere Untersuchung, welche ich mir vorbehalte, genauer hierüber Auskunft geben. Ebenso möchte ich die Frage, ob und welche Cometen der friheren Jahrhunderte in unser System hineinpassen, vorläufig als eine offene betrachten.

Kiel 1886 Febr. 12. 